\title{
Design of Fractional Order Recursive Digital Differintegrators using Different Approximation Techniques
}

\author{
Madhu Jain \\ Department of Electronics and Communication Engineering, Jaypee Institute of Information Technology, \\ A-10, Sector-62, Noida 201307, Uttar Pradesh, India \\ E-mail: ermadhu2003@gmail.com \\ Maneesha Gupta \\ Advanced Electronics Lab, Division of Electronics and Communication Engineering, \\ Netaji Subhas University of Technology, Sector-3, Dwarka, New Delhi 110078, India \\ E-mail: maneeshapub@gmail.com
}

Received: 29 March 2019; Accepted: 12 August 2019; Published: 08 February 2020

\begin{abstract}
Digital integer and fractional order integrators and differentiators are very important blocks of digital signal processing. In many situations, integer order integrators and differentiators are not sufficient to model all kind of dynamics. For such systems, fractional order operators give better solution. This paper is based on design of a new family of fractional order integrators and differentiators using various approximation techniques. Here, digital fractional order integrators are designed by direct discretization method using different techniques like continued fraction expansion, Taylor series expansion, and rational Chebyshev approximation on the transfer function of Jain-Gupta-Jain second order integrator. Their response in frequency domain is compared. The frequency response of the proposed integrators with highest efficiency is also compared with the existing ones. It is proved that rational Chebyshev approximation based integrators have highest efficiency among them. The fractional order differentiators are also designed using proposed integrators. It is concluded that proposed family of fractional order operators show remarkable improvement in frequency response compared to all the existing ones over the entire Nyquist frequency range.
\end{abstract}

Index Terms - Fractional order digital differentiator and integrator, rational Chebyshev approximation, continued fraction expansion.

\section{INTRODUCTION}

Differintegrators is a term which is originated from the combination of differentiators and integrators together. Digital differentiators and integrators are two most important components of both integer order and fractional order calculus [1-21, 26]. These operators are the basic parts of many systems like signal processing, control, radar, sonar, communication, and medical applications. Integer order operators are useful for those systems which can be modeled using integral calculus. However, for various dynamic systems, integer order operators do not prove adequate to represent the characteristics accurately. For these types of systems, fractional order operators prove more useful as compared to the integer order ones [8-22].

The interest of these fractional order operators in signal processing applications have been motivated by their good performances and robustness in many applications. In addition, the generalization of derivatives and integrals from integer orders to fractional orders gives more flexibility in designing signal processing algorithms.

This paper focuses on Taylor series expansion (TSE) $[11,15]$, continued fraction expansion (CFE) [8, 14, 15, 16, 18], and rational Chebyshev approximation (RCA) $[22,23,24,25]$ based realization of a family of fractional order $(\alpha)$ differintegrators (FODIs) where $\alpha \in[0.1,0.9]$ using Jain-Gupta-Jain second order integrator [3] by applying direct discretization method. The method using TSE and CFE expansion has been previously used by researchers in designing of FODIs for mostly $\alpha=0.5$. These are mainly based on first order operators and their results could not approximate the magnitude and phase characteristics of the ideal ones efficiently and even some of them have high computational complexity.

Rest of the paper is organized as follows. Section II presents the related works. Section III gives the problem formulation and solution methodology. Section IV presents the design of proposed FOIs and their comparison with the existing ones. Section V presents the proposed FODs and their comparison with the existing ones. The conclusions are given in section VI. Here, software MATLAB 8.0 is used to derive all simulation results and Nyquist frequency is taken as $\pi$ radians/ second. 


\section{RELATED WORKS}

In the literature, several definitions are given for arbitrary order differentiation and integration. The two most well-known definitions are the Riemann-Liouville (RL) and the Grunwald-Letnikov (GL).

RL definition $(\alpha>0)$ :

$$
{ }_{a} \mathrm{D}_{\mathrm{t}}^{\alpha} \mathrm{f}(\mathrm{t})=\frac{1}{\Gamma(\mathrm{n}-\alpha)} \frac{\mathrm{d}^{\mathrm{n}}}{\mathrm{dt}^{\mathrm{n}}} \int_{a}^{\mathrm{t}} \frac{\mathrm{f}(\tau) \mathrm{d} \tau}{(\mathrm{t}-\tau)^{\alpha-\mathrm{n}+1}}
$$

where $(\mathrm{n}-1)<\alpha<\mathrm{n}$ and $\Gamma(\mathrm{x})$ represents the Gamma function of $\mathrm{x}$.

GL definition $(\alpha \varepsilon \mathrm{R})$ :

$$
\begin{gathered}
{ }_{a} \mathrm{D}_{\mathrm{t}}^{\alpha} \mathrm{f}(\mathrm{t})=\lim _{\mathrm{h}_{0} \rightarrow 0} \frac{1}{\mathrm{~h}_{0}{ }^{\alpha}} \sum_{\mathrm{k}=0}^{\left[\mathrm{t}-a / \mathrm{h}_{0}\right]}(-1)^{\alpha}\left(\begin{array}{l}
\alpha \\
\mathrm{k}
\end{array}\right) \mathrm{f}\left(\mathrm{t}-\mathrm{kh}_{0}\right) \\
\left(\begin{array}{l}
\alpha \\
k
\end{array}\right)=\frac{\Gamma(\alpha+1)}{\Gamma(k+1) \Gamma(\alpha-k+1)}
\end{gathered}
$$

where $\mathrm{h}_{0}$ is time increment. As mentioned, the GL definition is valid for $\alpha>0$ (fractional differentiator) and for $\alpha<0$ (fractional integrator). From the perspective of control and signal processing applications, GL definition seems to be more useful, particularly in digital implementation.

One of important research topics of digital signal processing is to design digital fractional order differentiators (FODs) and fractional order integrators (FOIs).

The most popular methods are direct and indirect discretization using series expansion like continued fraction $[8,14,15,16]$, Taylor series $[11,15]$, and power series [12, 18], Pade's approximations [9, 20], Prony's approximations [9], Shank's approximations [9], least squares approximations [10], weighted least square approximations [10], radial basis function [13], and impulse invariant discretization techniques [17]. In direct discretization method, a generating function is expanded by direct application of an expansion series. On the other hand, in indirect discretization method an efficiently fitted $s$ domain rational approximation is discretized by using any existing $\mathrm{s}$ to $\mathrm{z}$ transformation.

\section{PROBLEM Formulation AND SOLUTION METHODOLOGIES}

Integer order operators are useful for those systems which can be modeled using integral calculus. However, for various dynamic systems, integer order operators do not prove adequate to represent the characteristics accurately. It is well known that various existing lumped systems can be analyzed more accurately by fractional order systems as compared to the integer order ones. To effectively analyze complicated systems with fractional elements, it is necessary to develop approximations to the fractional operators using the standard integer order operators.

In literature, many algorithms have designed to improve the performance of digital FODIs. It is observed that getting efficient frequency response with low complexity is a difficult task.

In this paper, the main focus is to achieve efficiency of the ideal FODIs. For this, a family of new stable recursive FOIs is designed using three approximation techniques TSE, CFE, and RCA. Subsequently, by modifying the transfer function of FOIs appropriately, new stable FODs are obtained.

The order of the designed FODIs is basically an arrangement between the superior performance in frequency domain (obtained by increased number of coefficients) and better hardware implementation (obtained by lower number of coefficients). In this work, the maximum order of the resultant FODIs are restricted to five only.

\section{PROPOSED FractionAl ORDER INTEGRATORS}

In this Section, the transfer function of Jain-Gupta-Jain second order integrator [3] is expanded for fractional powers using three expansion methods like TSE, CFE, and RCA. Then, the approximated mathematical models are derived by collecting the coefficients of the numerator and denominator polynomials. It is expected to obtain the designed FODIs with better accuracy.

The design process can be summarized in the following two steps:

(i) Discretize the s- domain fractional order integrator using a suitable generating function $(1 / \mathrm{s})^{\alpha}=\left(\mathrm{H}\left(\mathrm{z}^{-1}\right)\right)^{\alpha}$ where $\alpha$ denotes the fractional order, $\alpha \in[0.1,0.9]$.

(ii) Obtain the equivalent fractional order integrator, by performing TSE, CFE and RCA approximations over $\left(\mathrm{H}\left(\mathrm{z}^{-1}\right)\right)^{\alpha}$.

Here, expansion of $\left.\left(\mathrm{H}_{(\mathrm{z}}^{-1}\right)\right)^{\alpha}$ is an infinite order of rational discrete-time transfer function. In this paper, the order of the approximate mathematical model of the fractional order integrators is taken as 5 .

\section{A. Fractional Order Integrators via Taylor series expansion}

A family of FOIs is designed using Jain-Gupta-Jain second order integrator $\mathrm{H}_{\mathrm{JGJ}}\left(\mathrm{z}^{-1}\right)$ [3] and TSE. The transfer function of $\mathrm{H}_{\mathrm{JGJ}}\left(\mathrm{z}^{-1}\right)(4)$ is taken as a generating function.

$$
\mathrm{H}_{\mathrm{JGJ}}\left(\mathrm{z}^{-1}\right)=\frac{T\left(0.8647+0.5998 \mathrm{z}^{-1}+0.0541 \mathrm{z}^{-2}\right)}{\left(1-0.4812 \mathrm{z}^{-1}-0.5142 \mathrm{z}^{-2}\right)}
$$

So, the transfer function of FOIs for $\alpha \in[0.1,0.9]$ based on $\mathrm{H}_{\mathrm{JGJ}}\left(\mathrm{Z}^{-1}\right)$ can be expressed as: 


$$
\begin{aligned}
& \left(\mathrm{H}_{\mathrm{JGJ}}\left(\mathrm{z}^{-1}\right)\right)^{\alpha}=\mathrm{H}_{\mathrm{JGJ}}^{\alpha}\left(\mathrm{z}^{-1}\right) \\
& =\left(\frac{T\left(0.8647+0.5998 \mathrm{z}^{-1}+0.0541 \mathrm{z}^{-2}\right)}{\left(1-0.4812 \mathrm{z}^{-1}-0.5142 \mathrm{z}^{-2}\right)}\right)^{\alpha}
\end{aligned}
$$

Clearly, $\left(\mathrm{H}_{\mathrm{JGJ}}\left(\mathrm{z}^{-1}\right)\right)^{\alpha}$ is an infinite order of rational discrete-time transfer function. Here, MATLAB software is used to obtain the models of FOIs and series is truncated for fifth order polynomials (degree of the numerator and denominator is taken as 5).

The resultant transfer functions are unstable as poles are located outside the unit circle, these are stabilized by using pole reflection and magnitude compensation method suggested in [26]. After stabilization, the transfer function of the TSE deigned FOIs are shown as $\mathrm{H}_{\mathrm{TSE}, \alpha}\left(\mathrm{z}^{-1}\right)$ where $\alpha \in[0.1,0.9]$, these are given in (6-14).

$$
\begin{aligned}
\mathrm{H}_{\mathrm{TSE}, 0.1}\left(z^{-1}\right)= & \frac{\left.0.5604 \mathrm{e} 2 z^{-3}-0.8238 \mathrm{e} 2 z^{-4}+0.1094 \mathrm{e} 2 z^{-5}\right)}{\left(1-0.2548 \mathrm{e} 1 z^{-1}-0.6184 \mathrm{e} 1 z^{-2}-0.2544 \mathrm{e} 1 z^{-3}\right.}(6) \\
& \left.-0.1052 \mathrm{e} 1 z^{-4}-0.1603 \mathrm{e} 1 z^{-5}\right)
\end{aligned}
$$$$
\mathrm{H}_{\mathrm{TSE}, 0.2}\left(z^{-1}\right)=\frac{\begin{array}{l}
0.9450\left(1+0.1387 z^{-1}-0.2597 \mathrm{e} 1 z^{-2}\right. \\
+
\end{array}}{} \begin{aligned}
& \left(1257 \mathrm{e} 2 z^{-3}-0.9624 \mathrm{e} 1 z^{-1}-0.1214 z^{-2}-0.4494 \mathrm{e} 1 z^{-3}\right. \\
& \left.-0.4010 \mathrm{e} 1 z^{-4}-0.2668 \mathrm{e} 1 z^{-5}\right)
\end{aligned}
$$$$
\mathrm{H}_{\mathrm{TSE}, 0.3}\left(z^{-1}\right)=\frac{\begin{array}{l}
0.9187\left(1+0.2081 z^{-1}-0.3174 \mathrm{e} 1 z^{-2}\right. \\
\left.+0.0131 \mathrm{e} 1 z^{-3}-0.9543 \mathrm{e} 2 z^{-4}+0.1901 z^{-5}\right)
\end{array}}{\begin{array}{l}
\left(1-0.1444 z^{-1}-0.1786 z^{-2}-0.5859 \mathrm{e} 1 z^{-3}\right. \\
\left.-0.5117 \mathrm{e} 1 z^{-4}-0.3268 \mathrm{e} 1 z^{-5}\right)
\end{array}}
$$$$
0.8903\left(1+0.2775 z^{-1}-0.3270 \mathrm{e} 1 z^{-2}\right.
$$$$
\mathrm{H}_{\mathrm{TSE}, 0.4}\left(z^{-1}\right)=\frac{\left.+0.0109 \mathrm{e} 1 z^{-3}-0.4318 \mathrm{e} 2 z^{-4}+0.1855 \mathrm{e} 2 z^{-5}\right)}{\left(1-0.1592 z^{-1}-0.1825 z^{-2}-0.4596 \mathrm{e} 1 z^{-3}\right.}
$$$$
\left.-0.3899 \mathrm{e} 1 z^{-4}-0.1434 \mathrm{e} 1 z^{-5}\right)
$$$$
\mathrm{H}_{\mathrm{TSE}, 0.5}\left(z^{-1}\right)=\frac{\begin{array}{l}
0.8796\left(1+0.3468 z^{-1}-0.2884 \mathrm{e} 1 z^{-2}\right. \\
\left.+0.1000 \mathrm{e} 1 z^{-3}-0.3886 \mathrm{e} 2 z^{-4}+0.1636 \mathrm{e} 2 z^{-5}\right)
\end{array}}{\left(\begin{array}{l}
\left(1-0.2406 z^{-1}-0.2598 z^{-2}-0.4768 \mathrm{e} 1 z^{-3}\right. \\
\left.-0.5747 \mathrm{e} 1 z^{-4}-0.13351 \mathrm{e} 1 z^{-5}\right)
\end{array}\right.}
$$$$
\mathrm{H}_{\mathrm{TSE}, 0.6}\left(z^{-1}\right)=\frac{\begin{array}{l}
0.8402\left(1+0.400 \mathrm{l}^{-1}-.0128 \mathrm{e} 1 z^{-2}\right. \\
\left.+0.8269 \mathrm{e} 2 z^{-3}-0.3189 \mathrm{e} 2 z^{-4}+0.1318 \mathrm{e} 2 z^{-5}\right)
\end{array}}{\begin{array}{l}
\left(1-0.2887 z^{-1}-0.2830 z^{-2}-0.6562 \mathrm{e} 1 z^{-3}\right. \\
\left.-0.5353 \mathrm{e} 1 z^{-4}-0.2966 \mathrm{e} 1 z^{-5}\right)
\end{array}}
$$

$$
\mathrm{H}_{\mathrm{TSE}, 0.7}\left(z^{-1}\right)=\frac{\begin{array}{l}
0.8410\left(1+0.4856 z^{-1}-0.6701 \mathrm{e} 2 z^{-2}\right. \\
\left.+0.6067 \mathrm{e} 2 z^{-3}-0.9688 \mathrm{e} 2 z^{-4}+0.9575 \mathrm{e} 3 z^{-5}\right)
\end{array}}{\left(1-0.3368 z^{-1}-0.3843 z^{-2}-0.5703 \mathrm{e} 1 z^{-3}\right.}
$$$$
\mathrm{H}_{\mathrm{TSE}, 0.8}\left(z^{-1}\right)=\frac{\begin{array}{l}
0.8359\left(1+0.5549 z^{-1}+0.1159 \mathrm{e} 1 z^{-2}\right. \\
\left.+0.3732 \mathrm{e} 2 z^{-3}-0.1496 \mathrm{e} 2 z^{-4}+0.5989 \mathrm{e} 3 z^{-5}\right)
\end{array}}{\left(1-0.3578 z^{-1}-0.4029 z^{-2}-0.4315 \mathrm{e} 1 z^{-3}\right.}
$$

$$
\mathrm{H}_{\mathrm{TSE}, 0.9}\left(z^{-1}\right)=\frac{\begin{array}{l}
0.8372\left(1+0.6243 z^{-1}+0.3469 \mathrm{e} 1 z^{-2}\right. \\
\left.+0.1599 \mathrm{e} 2 z^{-3}-0.6908 \mathrm{e} 3 z^{-4}+0.2731 \mathrm{e} 3 z^{-5}\right)
\end{array}}{\left(1-0.433 z^{-1}-0.4732 z^{-2}-0.2411 \mathrm{e} 1 z^{-3}\right.}
$$

To define the efficiency of the designed FOIs, percentage absolute relative error (PARE) with respect to magnitude response is calculated in (15).

$$
\operatorname{PARE}(\omega)=\frac{\left|M_{\text {ideal }, \alpha}(\omega)-M_{T S E, \alpha}\left(e^{-j \omega}\right)\right|}{M_{\text {ideal }, \alpha}(\omega)} * 100
$$

Here, $M_{\text {ideal }, \alpha}(\omega)=\left|\left(\frac{1}{j \omega}\right)^{\alpha}\right|=$ amplitude of the ideal fractional order $(\alpha)$ integrator

$$
M_{T S E, \alpha}\left(e^{-j \omega}\right)=\left|H_{T S E, \alpha}\left(z^{-1}\right)\right|_{z=e^{j \omega}}=\text { amplitude of the }
$$

fractional order $(\alpha)$ integrator

The PARE response of TSE designed FOIs is shown in Fig. 1.

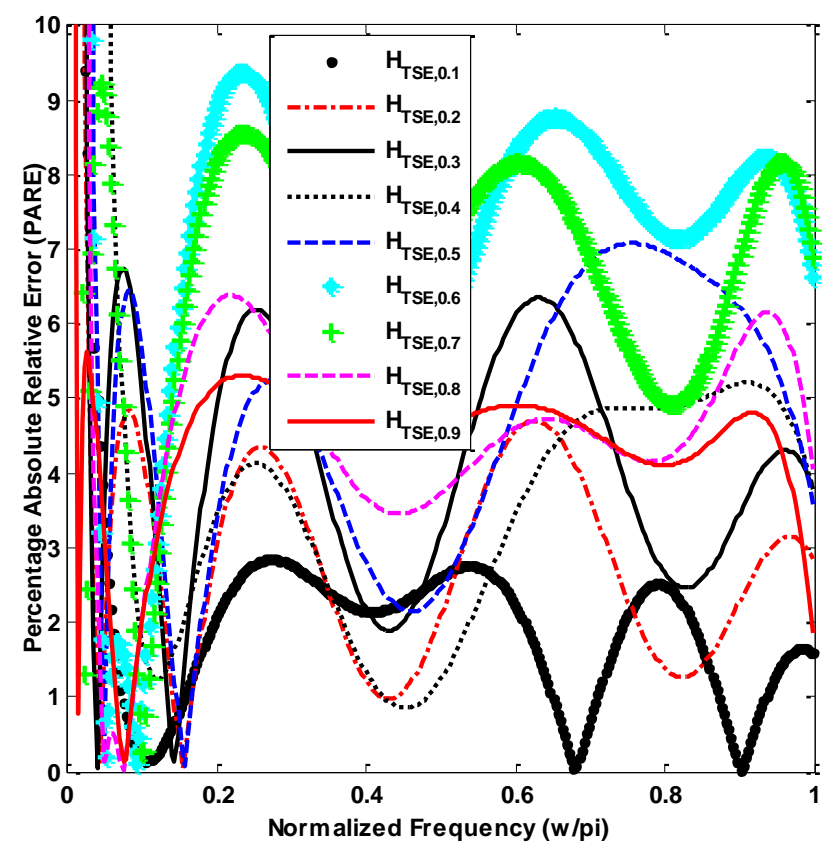

Fig.1. PARE response of TSE designed FOIs

Group delay is a measure of phase distortion and it is calculated by differentiating phase with respect to frequency. The degree of nonlinearity of the phase indicates the deviation of the group delay from a constant.

$$
\tau_{g}(\omega)=-\frac{d \theta(\omega)}{d \omega}
$$

Here phase of digital integrator is defined as $\theta(\omega)$. The group delay response of proposed FOIs is shown in Fig. 2. 


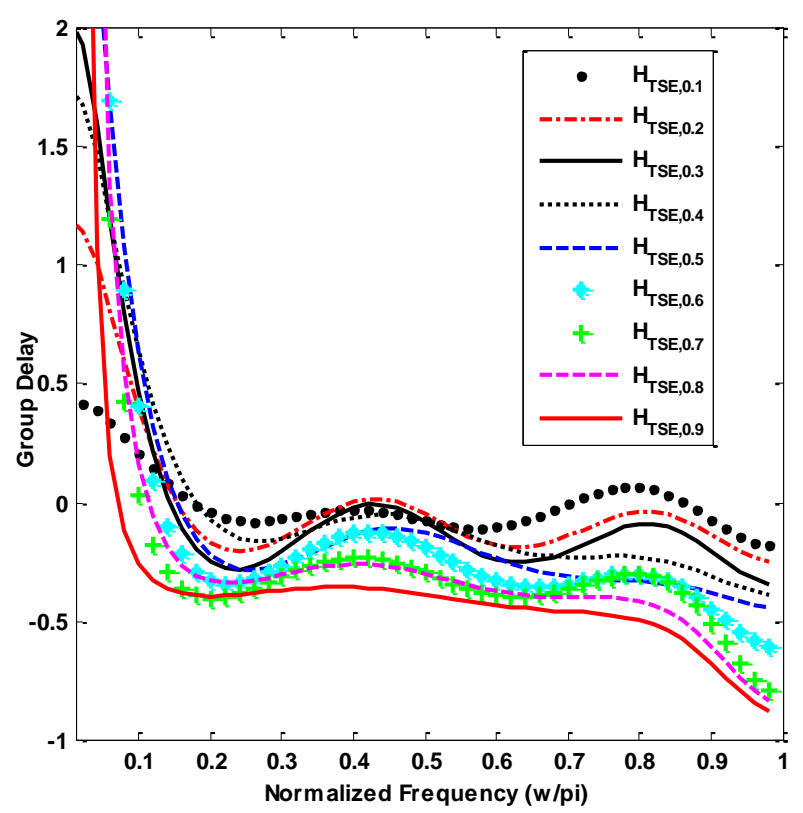

Fig.2. Group delay response of TSE designed FOIs

B. Fractional Order Integrators via Continued fraction expansion

In the second technique, $\left(\mathrm{H}_{\mathrm{JJJ}}\left(\mathrm{z}^{-1}\right)\right)^{\alpha}$ is expanded using CFE, and then depending on the order of the mathematical model required, the coefficients of the numerator and denominator polynomials are collected. In general, any function $\mathrm{G}(\mathrm{z})$ can be represented by continued fractions in the form of

$$
G(z) \approx a_{0}(z)+\frac{b_{1}(z)}{a_{1}(z)+\frac{b_{2}(z)}{a_{2}(z)+\frac{b_{3}(z)}{a_{3}(z)+\ldots \ldots . .}}}
$$

where the coefficients $a_{i}$ and $b_{i}$ are either rational functions of the variable $\mathrm{z}$ or constants. By truncation, an approximate rational function can be obtained. Here, transfer function of FOIs is obtained by collecting the coefficients of the numerator and the denominator polynomials for fifth order. The transfer function of CFE designed FOIs $\mathrm{H}_{\mathrm{CFE}, \mathrm{\alpha}}\left(\mathrm{z}^{-1}\right)$ are given in (19-27). The PARE and group delay response of CFE designed FOIs are shown in Fig. 3 and 4, respectively.

$$
\begin{aligned}
& \mathrm{H}_{\mathrm{CFE}, 0.1}\left(z^{-1}\right)=\left(\begin{array}{c}
-99.12-2018.12 z^{-1}+9306.23 z^{-2} \\
\frac{-0116.45 z^{-3}-2.87 \mathrm{e} 4 z^{-4}+2.20 \mathrm{e} 4 z^{-5}}{1-2753.65 z^{-1}+10220.1 z^{-2}+2285.32 z^{-3}}
\end{array}\right)
\end{aligned}
$$

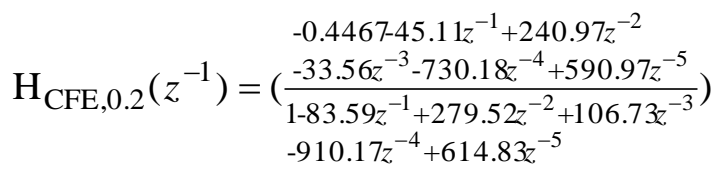

$$
\begin{aligned}
& \mathrm{H}_{\mathrm{CFE}, 0.3}\left(z^{-1}\right)=\left(\begin{array}{l}
-0.992317 .13 z^{-1}+106.05 z^{-2} \\
\begin{array}{l}
1-43.58 z^{-1}+131.82 z^{-2}+68.15 z^{-3} \\
-445.34 z^{-4}+291.41 z^{-5}
\end{array}
\end{array}\right. \\
& \mathrm{H}_{\mathrm{CFE}, 0.4}\left(z^{-1}\right)=\left(\begin{array}{c}
-0.66138 .38 z^{-1}+62.33 z^{-2} \\
\frac{-22.05 z^{-3}-190.41 z^{-4}+165.62 z^{-5}}{1-29.88 z^{-1}+82.12 z^{-2}+54.09 z^{-3}}
\end{array}\right) \\
& \mathrm{H}_{\mathrm{CFE}, 0.5}\left(z^{-1}\right)=\left(\frac{\begin{array}{l}
-0.5595-6.29 z^{-1}+44.14 z^{-2} \\
-23.26 z^{-3}-123.61 z^{-4}+117.23 z^{-5}
\end{array}}{1-22.93 z^{-1}+57.39 z^{-2}+46.35 z^{-3}}\right) \\
& -207.11 z^{-4}+126.25 z^{-5} \\
& \mathrm{H}_{\mathrm{CFE}, 0.6}\left(z^{-1}\right)=\left(\begin{array}{c}
-0.549-2.3189 z^{-1}+27.93 z^{-2} \\
1-18.71 z^{-1}+48.05 z^{-4}+83.02 z^{-5}
\end{array}\right) \\
& \mathrm{H}_{\mathrm{CFE}, 0.7}\left(z^{-1}\right)=\left(\begin{array}{c}
-0.26101 .45 z^{-1}+20.65 z^{-2} \\
\left.\begin{array}{l}
-17.98 z^{-3}-61.21 z^{-4}+65.78 z^{-5} \\
-129.03 z^{-4}+33.08 z^{-2}+37.55 z^{-3}
\end{array}\right)
\end{array}\right. \\
& -129.03 z^{-4}+73.52 z^{-5} \\
& \mathrm{H}_{\mathrm{CFE}, 0.8}\left(z^{-1}\right)=\left(\begin{array}{c}
-0.2920-36 z^{-1}+14.97 z^{-2} \\
\left.\begin{array}{l}
-15.66 z^{-3}-46.66 z^{-4}+52.11 z^{-5} \\
1-13.81 z^{-1}+26.31 z^{-2}+34.74 z^{-3}
\end{array}\right) \\
-107.52 z^{-4}+59.53 z^{-5}
\end{array}\right. \\
& \mathrm{H}_{\mathrm{CFE}, 0.9}\left(z^{-1}\right)=\left(\begin{array}{c}
-0.23980 .0216 z^{-1}+11.52 z^{-2} \\
\left(\begin{array}{l}
-14.07 z^{-3}-34.97 z^{-4}+42.97 z^{-5} \\
1-12.26 z^{-1}+21.33 z^{-2}+32.50 z^{-3}
\end{array}\right)
\end{array}\right.
\end{aligned}
$$

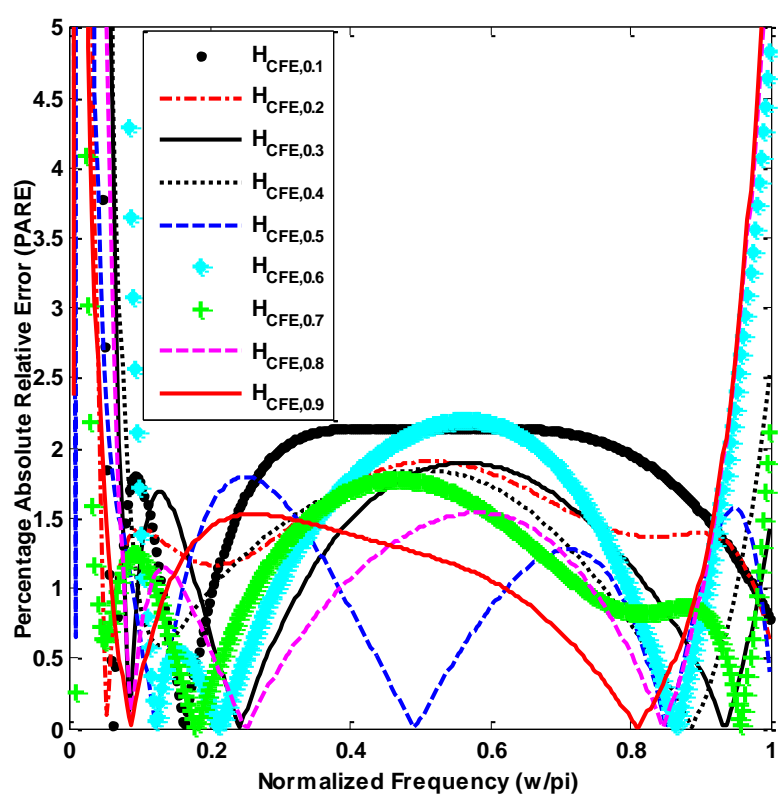

Fig.3. PARE response of CFE designed FOIs 


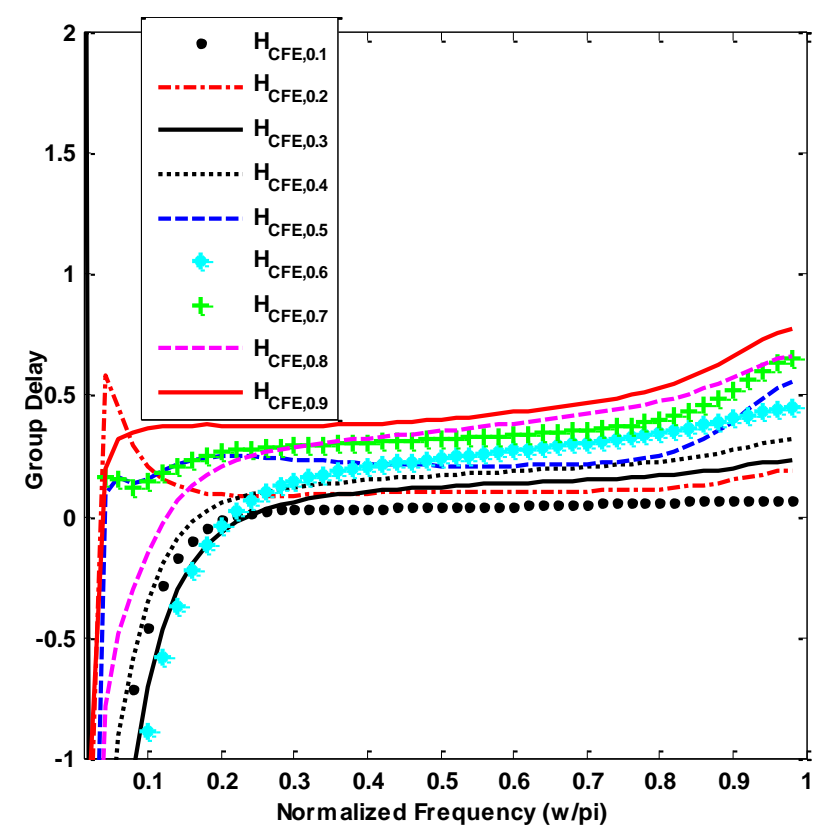

Fig.4. Group delay response of CFE designed FOIs

\section{Fractional Order Integrators via Rational Chebyshev} Approximation

In the third technique, $\left(\mathrm{H}_{\mathrm{JGJ}}\left(\mathrm{z}^{-1}\right)\right)^{\alpha}$ is expanded using rational Chebyshev approximation (RCA), and then depending on the order of the mathematical model required, the coefficients of the numerator and denominator polynomials are collected. It is well known that Chebyshev polynomials provide approximations very close to the true continuous functions due to their fast convergence properties.

The integrator is first expanded for fractional powers.

$$
\left(\mathrm{H}_{\mathrm{JGJ}}\left(\mathrm{z}^{-1}\right)\right)^{\alpha}=\mathrm{f}(\mathrm{z})
$$

Let the desired rational function be $\mathrm{H}_{\mathrm{PR}, \alpha}(\mathrm{z})$ which has a numerator of degree $m$ and a denominator of degree $n$.

$$
\mathrm{H}_{\mathrm{PR}, \alpha}\left(\mathrm{z}^{-1}\right)=\left(\frac{\mathrm{p}_{0}+\mathrm{p}_{1} \mathrm{z}^{-1}+\mathrm{p}_{2} \mathrm{z}^{-2}+\mathrm{p}_{3} \mathrm{z}^{-3}+\ldots \ldots \ldots \ldots \ldots \ldots \ldots+\mathrm{p}_{\mathrm{m}} \mathrm{z}^{-\mathrm{m}}}{1+\mathrm{q}_{1} \mathrm{z}^{-1}+\mathrm{q}_{2} \mathrm{z}^{-2}+\mathrm{q}_{3} \mathrm{z}^{-3}+\ldots \ldots \ldots \ldots \ldots \ldots \ldots+\mathrm{q}_{\mathrm{n}} \mathrm{z}^{-\mathrm{n}}}\right) \approx \mathrm{f}\left(\mathrm{z}^{-1}\right)
$$

Here, $\mathrm{p}_{0}, \mathrm{p}_{1}, \mathrm{p}_{2, \ldots} \ldots \mathrm{p}_{\mathrm{m}}$ and $\mathrm{q}_{1}, \mathrm{q}_{2, \ldots} \quad \mathrm{q}_{\mathrm{n}}$ are $(\mathrm{m}+\mathrm{n}+1)$ unknown parameters. Let $\lambda\left(z^{-1}\right)$ is the deviation of

$\mathrm{H}_{\mathrm{PR}, \mathrm{r}}\left(\mathrm{z}^{-1}\right)$ from $\mathrm{f}\left(\mathrm{z}^{-1}\right)$, and $\lambda$ is its maximum absolute value,

$$
\begin{gathered}
\lambda\left(\mathrm{z}^{-1}\right) \equiv \mathrm{H}_{\mathrm{PR}, \alpha}\left(\mathrm{z}^{-1}\right)-\mathrm{f}\left(\mathrm{z}^{-1}\right) \\
\lambda \equiv \max \left|\lambda\left(\mathrm{z}^{-1}\right)\right|
\end{gathered}
$$

The ideal minimax solution would be that choice of p's and q's that minimizes $\lambda$. It is well known that finding a rational function approximation is not as straight forward as finding a polynomial approximation. The interval of approximation is considered as $[a, b]$.
If $\mathrm{H}_{\mathrm{PR}, \alpha}\left(\mathrm{z}^{-1}\right)$ has no common polynomial factors in numerator and denominator, then there is a unique choice of p's and q's that minimizes $\lambda$; for this choice, $\lambda\left(z^{-1}\right)$ has $(\mathrm{m}+\mathrm{n}+2)$ extrema in $a \leq \mathrm{z} \leq b$, as mentioned by Ralston in [22]. Instead of making $\mathrm{f}\left(\mathrm{z}_{\mathrm{i}}^{-1}\right)$ and $\mathrm{H}_{\mathrm{PR}, \alpha}\left(\mathrm{z}_{\mathrm{i}}^{-1}\right)$ equal at some $(m+n+1)$ points $z_{i}^{-1}$, the residual $\lambda\left(z_{i}^{-1}\right)$ can be forced to any desired value $y_{i}$ [23]. Remez algorithms [22, 24], based on Chebyshev polynomials theory, explain the process of convergence by an iterative process. Some of these algorithms are easily convertible to computer programs as suggested in $[22,25]$. Here, we are using the algorithm proposed in [23] to find the optimal values of p's and q's. They provide the source code in $\mathrm{C}$ which can be easily used. Here, RCA is approximated in the interval [-0.9999, 0.9999] and degree of the numerator and denominator is taken as 5 .

The transfer function of RCA designed FOIs are defined as $\mathrm{H}_{\mathrm{FOI}, \alpha}\left(\mathrm{z}^{-1}\right)$, given in (31-39).

The PARE and group delay response of RCA designed FOIs are shown in Fig. 5 and 6, respectively.

$$
\begin{aligned}
& \mathrm{H}_{\mathrm{FOI}, 0.1}\left(z^{-1}\right)=\left(\frac{\begin{array}{l}
-0.9867+1.2769 z^{-1}+0.0041 z^{-2} \\
-0.4146 z^{-3}+0.0885 z^{-4}+0.0023 z^{-5}
\end{array}}{-0.1185 z^{-4}+0.0000 z^{-5}}\right) \\
& \mathrm{H}_{\mathrm{FOI}, 0.2}\left(z^{-1}\right)=\left(\frac{\begin{array}{l}
-0.9724+1.2011 z^{-1}+0.0554 z^{-2} \\
-0.3964 z^{-3}+0.0742 z^{-4}+0.0030 z^{-5}
\end{array}}{\begin{array}{l}
1.00001 .4709 z^{-1}+0.1709 z^{-2}+0.4473 z^{-3} \\
-0.1338 z^{-4}+0.0016 z^{-5}
\end{array}}\right. \\
& \mathrm{H}_{\mathrm{FOI}, 0.3}\left(z^{-1}\right)=\left(\frac{\begin{array}{l}
-0.9563+1.1237 z^{-1}+0.1028 z^{-2} \\
-0.3753 z^{-3}+0.0606 z^{-4}+0.0035 z^{-5}
\end{array}}{-0.1495 z^{-4}+0.0034 z^{-5}}\right)
\end{aligned}
$$

$$
\mathrm{H}_{\mathrm{FOI}, 0.4}\left(z^{-1}\right)=\left(\frac{\begin{array}{l}
-0.9448+1.0542 z^{-1}+0.1453 z^{-2} \\
1.000001 .5852 z^{-3}+0.0487 z^{-1}+0.2995 z^{-2}+0.454 z^{-5}
\end{array}}{-0.1655 z^{-4}+0.0054 z^{-5}}\right)
$$

$$
\mathrm{H}_{\mathrm{FOI}, 0.5}\left(z^{-1}\right)=\left(\begin{array}{c}
\frac{-0.9311+0.9809 z^{-1}+0.1851 z^{-2}}{1.00001 .6337 z^{-3}+0.0362 z^{-4}+0.004 z^{-5}+0.3680 z^{-2}+0.4554 z^{-3}} \\
-0.1817 z^{-4}+0.0079 z^{-5}
\end{array}\right)
$$

$$
\mathrm{H}_{\mathrm{FOI}, 0.6}\left(z^{-1}\right)=\left(\begin{array}{c}
-0.9187+0.9131 z^{-1}+0.2204 z^{-2} \\
\left.\begin{array}{l}
-0.3127 z^{-3}+0.0275 z^{-4}+0.0039 z^{-5} \\
-0.1986 z^{-4}+0.0106 z^{-5}
\end{array}\right)
\end{array}\right.
$$

$$
\mathrm{H}_{\mathrm{FOI}, 0.7}\left(z^{-1}\right)=\left(\begin{array}{c}
-0.9016+0.8405 z^{-1}+0.2507 z^{-2} \\
\left.\begin{array}{l}
-0.2887 z^{-3}+0.0184 z^{-4}+0.0037 z^{-5} \\
-0.2158 z^{-4}+0.0136 z^{-5}
\end{array}\right)
\end{array}\right.
$$

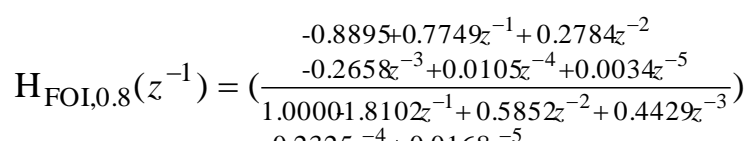




$$
\mathrm{H}_{\mathrm{FOI}, 0.9}\left(z^{-1}\right)=\left(\begin{array}{c}
-0.8771+0.7089 z^{-1}+0.3029 z^{-2} \\
\left.\begin{array}{l}
-0.2424 z^{-3}+0.0036 z^{-4}+0.0030 z^{-5} \\
-0.24901 .8650 z^{-4}+0.0203 z^{-5}
\end{array}\right)
\end{array}\right.
$$

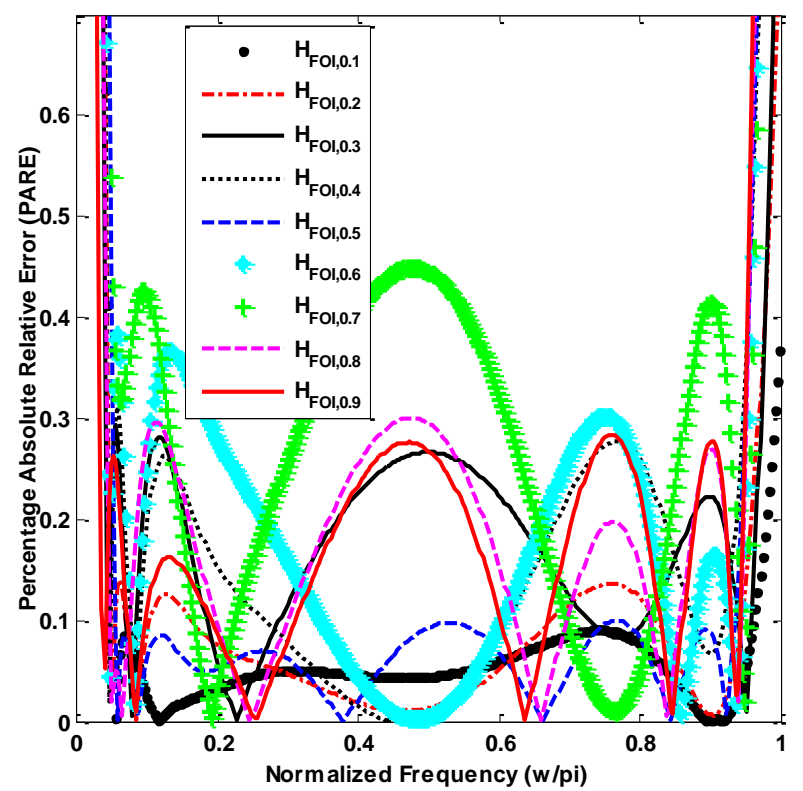

Fig.5. PARE response of RCA designed FOIs

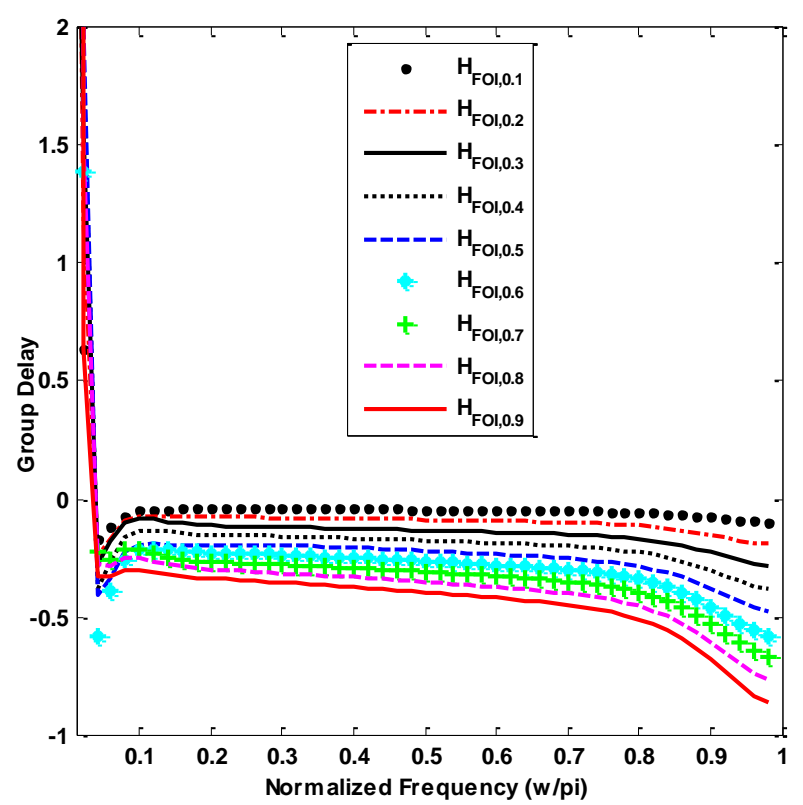

Fig.6. Group delay response of RCA designed FOIs

D. Comparison of Designed Fractional Order Integrators with the existing FOIs

By observing the frequency response (Fig. 1-6), it can be seen that RCA provides much better approximation than TSE and CFE. The pole-zero distribution of the proposed RCA designed FOIs are shown in Table 1. It can be observed that all of these have poles and zeros inside the unit circle, ensuring their stability.
Table 1. Pole- Zero Distribution of Proposed RCA Designed Fractional Order Integrators

\begin{tabular}{|c|c|c|}
\hline $\begin{array}{c}\text { RCA } \\
\text { designed } \\
\text { FOIs }\end{array}$ & Zeros & Poles \\
\hline \multirow{2}{*}{$\mathrm{H}_{\mathrm{FOI}, 0.1}\left(\mathrm{z}^{-1}\right)$} & $-0.5577,0.6601,0.9220$, & $-0.5504,0.6916,0.9407$, \\
& $0.2929,-0.0231$ & $0.3306,+3.6377 \mathrm{e}-004$ \\
\hline \multirow{2}{*}{$\mathrm{H}_{\mathrm{FOI}, 0.2}\left(\mathrm{z}^{-1}\right)$} & $-0.5612,0.6431,0.9137$, & $-0.5470,0.7087,0.9479$, \\
& $0.2739,-0.0343$ & $0.3488,0.0125$ \\
\hline \multirow{2}{*}{$\mathrm{H}_{\mathrm{FOI}, 0.3}\left(\mathrm{z}^{-1}\right)$} & $-0.5643,0.6231,0.9066$, & $-0.5435,0.7246,0.9551$, \\
& $0.2548,-0.0452$ & $0.3674,0.0248$ \\
\hline \multirow{2}{*}{$\mathrm{H}_{\mathrm{FOI}, 0.4}\left(\mathrm{z}^{-1}\right)$} & $-0.5677,0.6083,0.8953$, & $-0.5397,0.7407,0.9615$, \\
& $0.2356,-0.0557$ & $0.3855,0.0374$ \\
\hline \multirow{2}{*}{$\mathrm{H}_{\mathrm{FOI}, 0.5}\left(\mathrm{z}^{-1}\right)$} & $0.2133,0.5958,0.8821$, & $-0.5357,0.4024,0.7575$, \\
& $-0.5695,-0.0682$ & $0.9685,0.0502$ \\
\hline \multirow{2}{*}{$\mathrm{H}_{\mathrm{FOI}, 0.6}\left(\mathrm{z}^{-1}\right)$} & $0.1965,0.5714,0.8757$, & $-0.5311,0.4223,0.7698$, \\
& $-0.5744,-0.0753$ & $0.9775,0.0629 ;$ \\
\hline \multirow{2}{*}{$\mathrm{H}_{\mathrm{FOI}, 0.7}\left(\mathrm{z}^{-1}\right)$} & $0.1765,0.5534,0.8643$, & $-0.5283,0.4398,0.7893$, \\
& $-0.5777,-0.0842$ & $0.9785,0.0759$ \\
\hline \multirow{2}{*}{$\mathrm{H}_{\mathrm{FOI}, 0.8}\left(\mathrm{z}^{-1}\right)$} & $0.1563,0.5341,0.8540$, & $-0.5241,0.4585,0.8019$, \\
\hline \multirow{2}{*}{$\mathrm{H}_{\mathrm{FOI}, 0.9}\left(\mathrm{z}^{-1}\right)$} & $-0.5807,-0.0926$ & $0.9852,0.0887$ \\
\hline
\end{tabular}

It can be seen from Fig.5 that the proposed integrators; $\mathrm{H}_{\mathrm{FOI}, 0.1}\left(\mathrm{z}^{-1}\right)$ has PARE $\leq 0.08$ over $0.04 \pi \leq \omega \leq 0.97 \pi$ radians, $\mathrm{H}_{\mathrm{FOI}, 0.2}\left(\mathrm{z}^{-1}\right)$ has PARE $\leq 0.12$ over $0.04 \pi \leq \omega \leq$ $0.96 \pi$ radians, $\mathrm{H}_{\mathrm{FOI}, 0.3}\left(\mathrm{z}^{-1}\right)$ has $\mathrm{PARE} \leq 0.28$ over $0.03 \pi \leq$ $\omega \leq 0.98 \pi$ radians, $\mathrm{H}_{\mathrm{FOI}, 0.4}\left(\mathrm{z}^{-1}\right)$ has PARE $\leq 0.27$ over $0.04 \pi \leq \omega \leq 0.96 \pi$ radians, $\mathrm{H}_{\mathrm{FOI}, 0.5}\left(\mathrm{Z}^{-1}\right)$ has PARE $\leq 0.10$ over $0.05 \pi \leq \omega \leq 0.94 \pi$ radians, $\mathrm{H}_{\mathrm{FOI}, 0.6}\left(\mathrm{z}^{-1}\right)$ has PARE $\leq$ 0.38 over $0.04 \pi \leq \omega \leq 0.97 \pi$ radians, $\mathrm{H}_{\mathrm{FOI}, 0.7}\left(\mathrm{z}^{-1}\right)$ has PARE $\leq 0.42$ over $0.04 \pi \leq \omega \leq 0.97 \pi$ radians, $\mathrm{H}_{\mathrm{FOI}, 0.8}\left(\mathrm{z}^{-1}\right)$ has PARE $\leq 0.30$ over $0.03 \pi \leq \omega \leq 0.96 \pi$ radians and $\mathrm{H}_{\mathrm{FOI}, 0.9}\left(\mathrm{z}^{-1}\right)$ has PARE $\leq 0.27$ over $0.02 \pi \leq \omega \leq 0.96 \pi$ radians. To show efficiency of the proposed FOIs, various existing half order integrators have considered. These half order integrators are Krishna $\mathrm{H}_{\mathrm{K}, 0.5}\left(\mathrm{z}^{-1}\right)$ [14], Gupta-Varshney Visweswaran $\mathrm{H}_{\mathrm{GVV}, 0.5}\left(\mathrm{z}^{-1}\right)$ [15], GuptaJain-Jain $\mathrm{H}_{\mathrm{GJJ}, 0.5}\left(\mathrm{z}^{-1}\right)$ [16], and Li-Sheng-Chen $\mathrm{H}_{\mathrm{LSC}, 0.5}\left(\mathrm{z}^{-}\right.$ $\left.{ }^{1}\right)$ [17]. Their transfer functions are given in (40-43).

$$
\mathrm{H}_{\mathrm{GVV}, 0.5}\left(z^{-1}\right)=\frac{0.0224\left(1-0.8506 z^{-1}\right)\left(1-0.4708 z^{-1}\right)}{\left(1+0.2421 z^{-1}\right)\left(1-0.0391 z^{-1}\right)}
$$

$$
\mathrm{H}_{\mathrm{GJJ}, 0.5}\left(z^{-1}\right)=\frac{\begin{array}{l}
0.9309-0.9040 z^{-1}-0.4901 z^{-2} \\
+0.4421 z^{-3}+0.0726 z^{-4}-0.0366 z^{-5}
\end{array}}{1-1.5644 z^{-1}+0.0194 z^{-2}+0.7243 z^{-3}}
$$

$$
0.00167-0.006112 z^{-1}+0.00840 z^{-2}
$$

$$
\begin{aligned}
\mathrm{H}_{\mathrm{LSC}, 0.5}\left(z^{-1}\right)=\frac{-0.00520 \mathrm{z}^{-3}+0.00129 z^{-4}-4.785 \times 10^{-5} z^{-5}}{1-4.488 z^{-1}+8.004 z^{-2}-7.082 z^{-3}} \\
+3.104 z^{-4}-0.5383 z^{-5}
\end{aligned}
$$




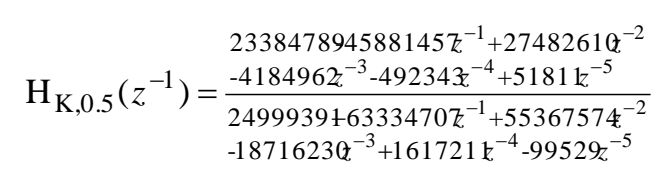

The PARE response and group delay response of the proposed half order integrator $(\alpha=0.5)$ with above mentioned existing half order integrators over Nyquist frequency range is shown in Fig. 7 and 8, respectively.

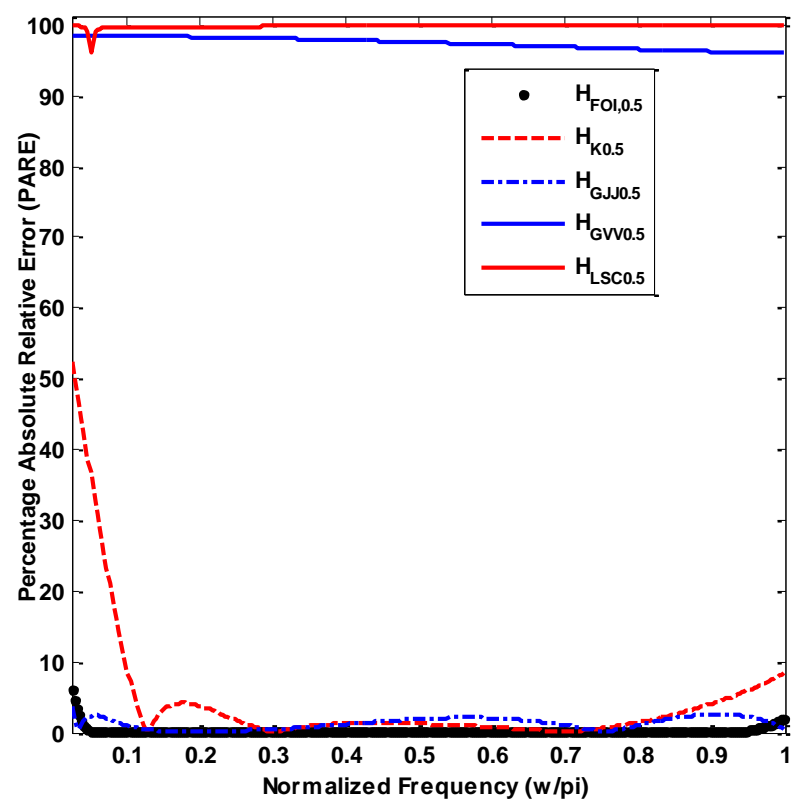

Fig.7. PARE response of proposed half order integrator $\mathrm{H}_{\mathrm{FOI}, 0.5}\left(\mathrm{z}^{-1}\right)$ and existing half order integrators; $\mathrm{H}_{\mathrm{K}, 0.5}\left(\mathrm{z}^{-1}\right)$ [14], $\mathrm{H}_{\mathrm{GVv}, 0.5}\left(\mathrm{z}^{-1}\right)$ [15], $\mathrm{H}_{\mathrm{GJ}, 0.5}\left(\mathrm{z}^{-1}\right)[16], \mathrm{H}_{\mathrm{LSC}, 0.5}\left(\mathrm{z}^{-1}\right)[17]$.

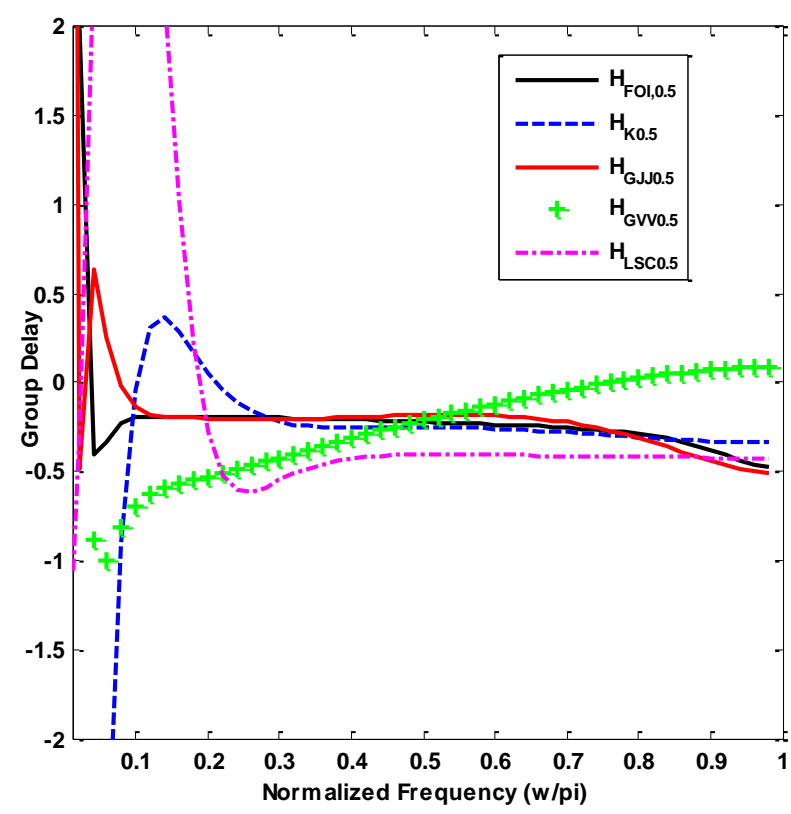

Fig.8. Group delay response of proposed half order integrator $\mathrm{H}_{\mathrm{FOI}, 0.5}\left(\mathrm{z}^{-1}\right)$ and existing half order integrators; $\mathrm{H}_{\mathrm{K}, 0.5}\left(\mathrm{z}^{-1}\right)$ [14], $\mathrm{H}_{\mathrm{GVV}, 0.5}\left(\mathrm{z}^{-1}\right)$ [15], $\mathrm{H}_{\mathrm{GJ}, 0.5}\left(\mathrm{z}^{-1}\right)[16], \mathrm{H}_{\mathrm{LSC}, 0.5}\left(\mathrm{z}^{-1}\right)[17]$.

It is verified from Fig. 7 that the proposed half order integrator $\mathrm{H}_{\mathrm{FOI}, 0.5}\left(\mathrm{z}^{-1}\right)$ has PARE $\leq 0.10$ over $0.05 \pi \leq \omega \leq$ $0.94 \pi$ radians while the existing half order integrator:
Krishna $\mathrm{H}_{\mathrm{K}, 0.5}\left(\mathrm{z}^{-1}\right)$ [14] has PARE $\leq 8.30$ over $0.11 \pi \leq \omega$ $\leq \pi$ radians, Gupta-Varshney-Visweswaran $\mathrm{H}_{\mathrm{GVV}, 0.5}\left(\mathrm{z}^{-1}\right)$ [15] has PARE $\leq 96$ over $0 \leq \omega \leq \pi$ radians, Gupta-JainJain $\mathrm{H}_{\mathrm{GJJ}, 0.5}\left(\mathrm{z}^{-1}\right)[16]$ has PARE $\leq 2.6$ over $0.30 \pi \leq \omega \leq \pi$ radians and Li- Sheng- Chen $\mathrm{H}_{\mathrm{LSC}, 0.5}\left(\mathrm{z}^{-1}\right)$ [17] has PARE $\leq 100$ over $0 \leq \omega \leq \pi$ radians.

It is verified from above results (Fig. 5 and 7) that proposed FOIs have very low PARE as compared to the existing ones over the entire Nyquist frequency range. It is also confirmed from Fig. 6 and 8 that the proposed FOIs also have linear phase response as compared to the existing ones.

\section{Proposed Fractional ORdER DifFERENTIATORS AND THEIR COMPARISON WITH THE EXISTING FODS}

It is observed from Section IV that RCA gives better approximations than TSE and CFE. Here, FODs are obtained by inverting the transfer functions of RCA based FOIs $\mathrm{H}_{\mathrm{FOI}, \alpha}\left(\mathrm{z}^{-1}\right)$ (31-39) in the similar way as used in the design of analog differentiator by Al-Alaoui [26]. Their transfer functions $\mathrm{D}_{\mathrm{FOD}, \alpha}\left(\mathrm{z}^{-1}\right)$ are given in (44-52). The PARE and group delay response of these proposed FODs are shown in Fig. 9 and Fig. 10, respectively.

$$
\mathrm{D}_{\mathrm{FOD}, 0.1}\left(z^{-1}\right)=\left(\begin{array}{l}
1.00001 .4129 z^{-1}+0.1104 z^{-2} \\
\begin{array}{l}
-0.98400 z^{-3}-0.1185 z^{-4}+0.0000 z^{-5} \\
+0.0885 z^{-4}+0.00276 z^{-1}+0.004 z^{-5}
\end{array}
\end{array}\right.
$$

$$
\mathrm{D}_{\mathrm{FOD}, 0.2}\left(z^{-1}\right)=\left(\begin{array}{c}
1.00001 .4709 z^{-1}+0.1709 z^{-2} \\
\left.\begin{array}{l}
-0.4473 z^{-3}-0.1338 z^{-4}+0.0016 z^{-5} \\
+0.07421 .2011 z^{-4}+0.0030 z^{-5}
\end{array}\right)
\end{array}\right.
$$

$$
\mathrm{D}_{\mathrm{FOD}, 0.3}\left(z^{-1}\right)=\left(\begin{array}{c}
1.00001 .5284 z^{-1}+0.2339 z^{-2} \\
\frac{+0.4524 z^{-3}-0.1495 z^{-4}+0.0034 z^{-5}}{-0.9563+1.1237 z^{-1}+0.1028 z^{-2}-0.3753 z^{-3}}
\end{array}\right)
$$

$$
\mathrm{D}_{\mathrm{FOD}, 0.4}\left(z^{-1}\right)=\left(\begin{array}{c}
1.00001 .5854 z^{-1}+0.2995 z^{-2} \\
\left.\begin{array}{l}
+0.4549 z^{-3}-0.1655 z^{-4}+0.0054 z^{-5} \\
-0.9448+1.0542 z^{-1}+0.1453 z^{-2}-0.3562 z^{-3}
\end{array}\right) \\
+0.0487 z^{-4}+0.0038 z^{-5}
\end{array}\right.
$$

$$
\mathrm{D}_{\mathrm{FOD}, 0.5}\left(z^{-1}\right)=\left(\begin{array}{c}
1.00001 .6429 z^{-1}+0.3680 z^{-2} \\
\left.\begin{array}{l}
+0.4554 z^{-3}-0.1817 z^{-4}+0.0079 z^{-5} \\
+0.0362 z^{-4}+0.009 z^{-1}+0.1851 z^{-2}-0.3337 z^{-3}
\end{array}\right)
\end{array}\right.
$$$$
\mathrm{D}_{\mathrm{FOD}, 0.6}\left(z^{-1}\right)=\left(\begin{array}{c}
1.00001 .7014 z^{-1}+0.4412 z^{-2} \\
\left.\begin{array}{l}
+0.4525 z^{-3}-0.1986 z^{-4}+0.0106 z^{-5} \\
-0.9187+0.9131 z^{-1}+0.2204 z^{-2}-0.3127 z^{-3}
\end{array}\right) \\
+0.0275 z^{-4}+0.0039 z^{-5}
\end{array}\right.
$$

$$
\mathrm{D}_{\text {FOD }, 0.7}\left(z^{-1}\right)=\left(\begin{array}{c}
1.00001 .7552 z^{-1}+0.5110 z^{-2} \\
-0.4500 z^{-3}-0.2158 z^{-4}+0.0136 z^{-5} \\
-0.9016+0.8405 z^{-1}+0.2507 z^{-2}-0.2887 z^{-3}
\end{array}\right)
$$




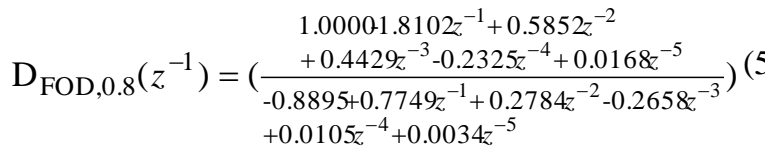

$$
\begin{aligned}
& \mathrm{D}_{\text {FOD }, 0.9}\left(z^{-1}\right)=\left(\frac{1.00001 .8650 z^{-1}+0.6610 z^{-2}}{-0.8771+0.7089 z^{-1}+0.302 z^{-4}+0.0203 z^{-2}-0.2424 z^{-3}}\right)(
\end{aligned}
$$

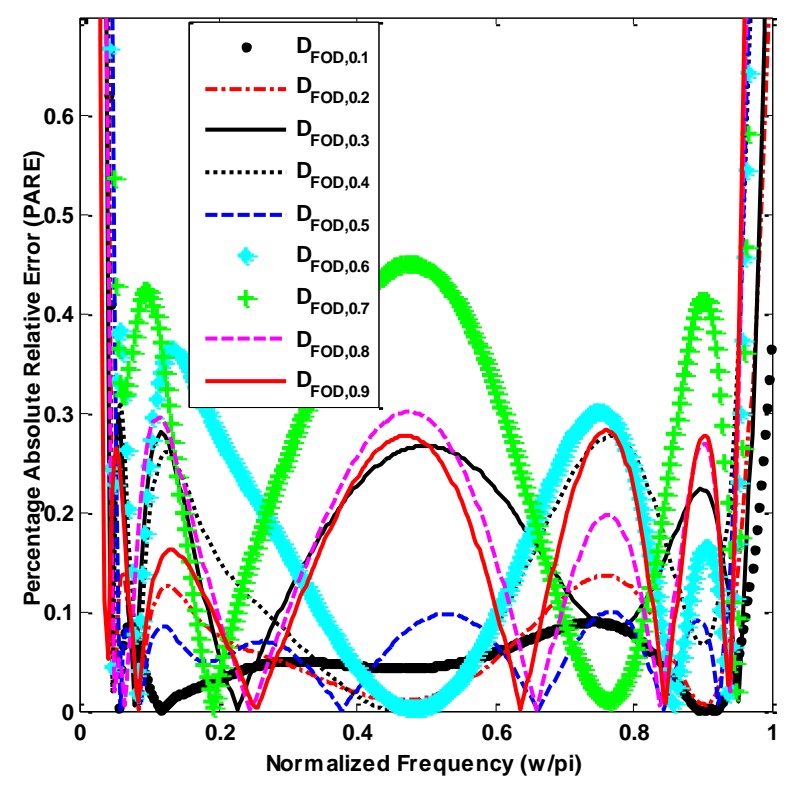

Fig.9. PARE response of proposed FODs

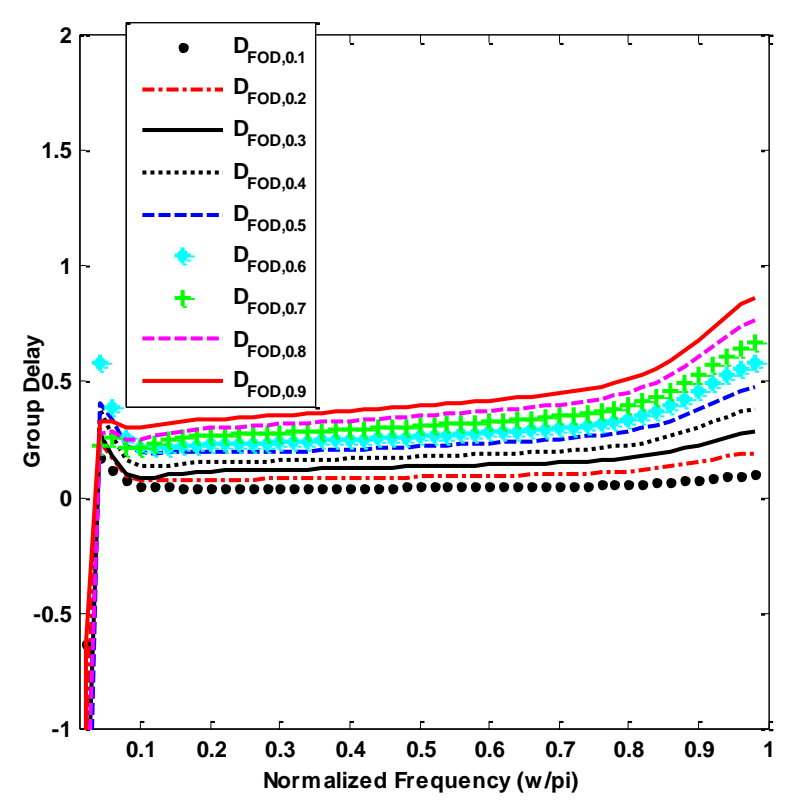

Fig.10. Group delay response of proposed FODs

It can be seen from Fig. 9 that the proposed fractional order differentiators; $\mathrm{D}_{\text {FOD }, 0.1}\left(\mathrm{z}^{-1}\right)$ has PARE $\leq 0.08$ over $0.04 \pi \leq \omega \leq 0.97 \pi$ radians, $D_{\mathrm{FOD}, 0.2}\left(\mathrm{Z}^{-1}\right)$ has PARE $\leq 0.12$ over $0.04 \pi \leq \omega \leq 0.96 \pi$ radians, $D_{\mathrm{FOD}, 0.3}\left(\mathrm{z}^{-1}\right)$ has PARE $\leq$ 0.28 over $0.03 \pi \leq \omega \leq 0.98 \pi$ radians, $\mathrm{D}_{\mathrm{FOD}, 0.4}\left(\mathrm{z}^{-1}\right)$ has
PARE $\leq 0.27$ over $0.04 \pi \leq \omega \leq 0.96 \pi$ radians, $\mathrm{D}_{\mathrm{FOD}, 0.5}\left(\mathrm{z}^{-1}\right)$ has PARE $\leq 0.10$ over $0.05 \pi \leq \omega \leq 0.94 \pi$ radians, $\mathrm{D}_{\mathrm{FOD}, 0.6}\left(\mathrm{z}^{-1}\right)$ has PARE $\leq 0.38$ over $0.04 \pi \leq \omega \leq 0.97 \pi$ radians, $\mathrm{D}_{\mathrm{FOD}, 0.7}\left(\mathrm{z}^{-1}\right)$ has PARE $\leq 0.42$ over $0.04 \pi \leq \omega \leq$ $0.97 \pi$ radians, $\mathrm{D}_{\mathrm{FOD}, 0.8}\left(\mathrm{z}^{-1}\right)$ has PARE $\leq 0.30$ over $0.03 \pi \leq$ $\omega \leq 0.96 \pi$ radians and $\mathrm{D}_{\mathrm{FOD}, 0.9}\left(\mathrm{z}^{-1}\right)$ has PARE $\leq 0.27$ over $0.02 \pi \leq \omega \leq 0.96 \pi$ radians.

To show the efficiency of the proposed FODs, various existing half order differentiators have been considered. These half order differentiators are Krishna $\mathrm{D}_{\mathrm{K}, 0.5}\left(\mathrm{z}^{-1}\right)$ [14], Leulmi-Ferdi $\mathrm{D}_{\mathrm{LFM}, 0.5}\left(\mathrm{z}^{-1}\right), \mathrm{D}_{\mathrm{LFT}, 0.5}\left(\mathrm{z}^{-1}\right)$ [18]. Their transfer functions are given in (53-55).

$$
\mathrm{D}_{\mathrm{K}, 0.5}\left(z^{-1}\right)=\frac{249993916333470 z^{-1}+5536757 \mathrm{q}^{-2}}{233847871623 \mathrm{Q}^{-3}+161721 \mathrm{z}^{-4}-99529 \mathrm{z}^{-5}}
$$

$\left(1.07590489205223 .0680788315322^{-1}+2.860311581957 z^{-2}\right.$ $\mathrm{D}_{\mathrm{LFM}, 0.5}\left(z^{-1}\right)=\frac{\left.-0.713214489179 z^{-3}-0.254818880361843480 z^{-5}-0.099911883438 \mathrm{q}^{-5}\right)}{\left(1.00000000000092 .261056172284 \mathrm{z}^{-1}+1.374906434448 \mathrm{z}^{-2}\right.}$
$\left.+0.108728794571 \mathrm{z}^{-3}-0.248348689526 z^{-4}+0.0262913 \oplus 1092 \mathrm{z}^{-5}\right)$

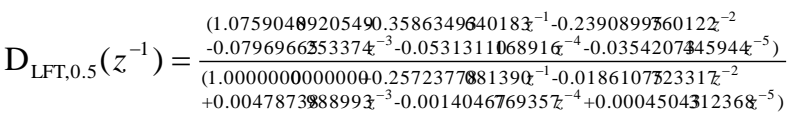

The PARE response and group delay response of the proposed half order differentiator $(\alpha=0.5)$ with above mentioned existing half order differentiators over Nyquist frequency range are shown in Fig. 11 and Fig. 12.

It is verified from Fig. 11 that the proposed half order differentiator $\mathrm{D}_{\mathrm{FOD}, 0.5}\left(\mathrm{z}^{-1}\right)$ has PARE $\leq 0.10$ over $0.05 \pi \leq$ $\omega \leq 0.94 \pi$ radians.

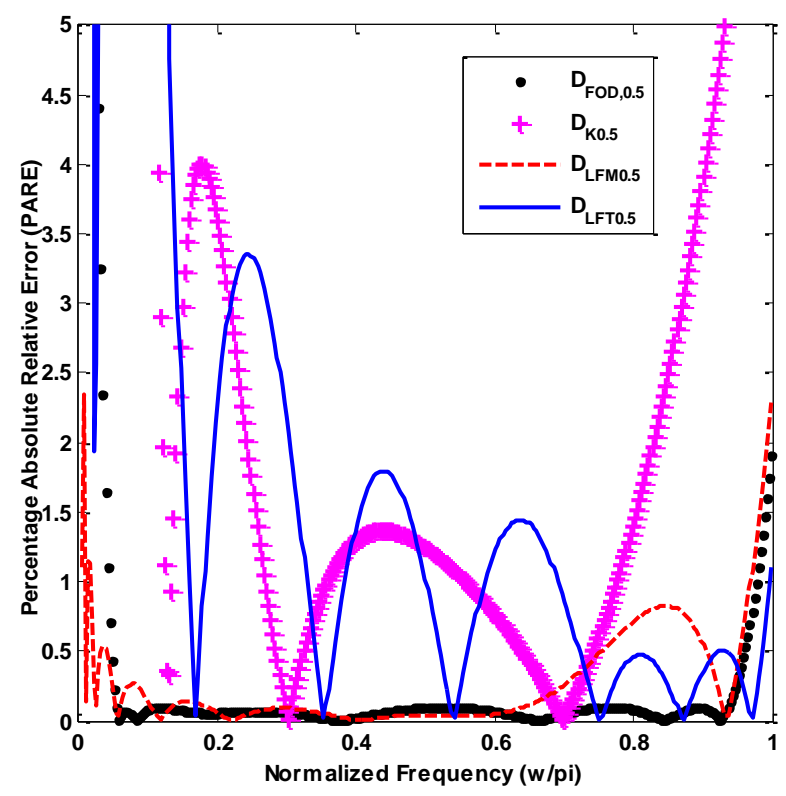

Fig.11. PARE response of proposed half order differentiator $\mathrm{D}_{\mathrm{FOD}, 0.5}\left(\mathrm{z}^{-1}\right)$ and existing half order differentiators; $\mathrm{D}_{\mathrm{K}, 0.5}\left(\mathrm{Z}^{-1}\right)$ [14], $\mathrm{D}_{\mathrm{LFM}, 0.5}\left(\mathrm{Z}^{-1}\right)[18]$, $\mathrm{D}_{\mathrm{LFT}, 0.5}\left(\mathrm{Z}^{-1}\right)[18]$ 


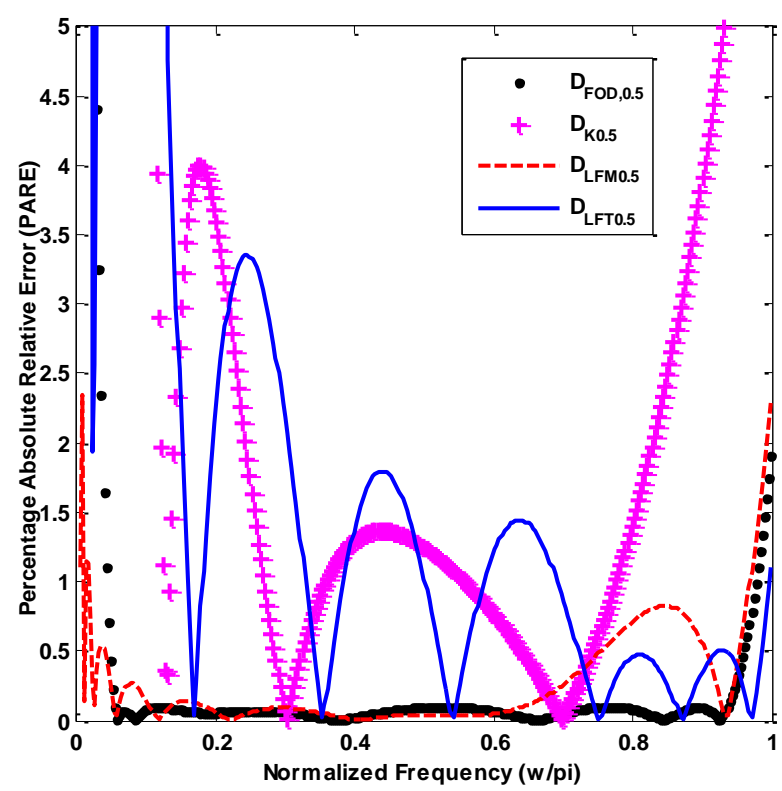

Fig.12. Group delay response of proposed half order differentiator $\mathrm{D}_{\mathrm{FOD}, 0.5}\left(\mathrm{z}^{-1}\right)$ and existing half order differentiators; $\mathrm{D}_{\mathrm{K}, 0.5}\left(\mathrm{Z}^{-1}\right)[14]$,

$$
\mathrm{D}_{\mathrm{LFM}, 0.5}\left(\mathrm{z}^{-1}\right)[18], \mathrm{D}_{\mathrm{LFT}, 0.5}\left(\mathrm{z}^{-1}\right)[18]
$$

The existing half order differentiators: Krishna $\mathrm{D}_{\mathrm{K}, 0.5}(\mathrm{z}$ $\left.{ }^{1}\right)$ [14] has PARE $\leq 7.66$ over $0.11 \pi \leq \omega \leq \pi$ radians, Leulmi-Ferdi $\mathrm{D}_{\mathrm{LFM}, 0.5}\left(\mathrm{z}^{-1}\right)$ [18] has PARE $\leq 2.24$ over $0.05 \pi \leq \omega \leq \pi$ radians and $\mathrm{D}_{\mathrm{LFT}, 0.5}\left(\mathrm{z}^{-1}\right)[18]$ has PARE $\leq$ 4.84 over $0.13 \pi \leq \omega \leq \pi$ radians.

It is seen from Figs. 9 and 11 that proposed FODs have very low PARE compared to the existing ones over entire Nyquist frequency range. It is also observed from Figs. 10 and 12 that the proposed FODs have linear phase response compared to the Krishna's half order differentiator $\mathrm{D}_{\mathrm{K}, 0.5}\left(\mathrm{z}^{-1}\right)$ [14] and Leulmi- Ferdi's half order differentiator $\mathrm{D}_{\mathrm{LFT}, 0.5}\left(\mathrm{z}^{-1}\right)$ [18].

\section{CONCLUSION}

In this paper, design and analysis of digital fractional order differintegrators are discussed. The digital fractional order integrators are designed by direct discretization method using TSE, CFE, and rational Chebyshev approximation. Jain-Gupta-Jain second order integrator is used as a generating function. After that, by modifying these transfer functions fractional order differentiators are designed. The best result is obtained for 0.1 order integrator and differentiator with PARE $\leq$ 0.08 over the frequency range $0.04 \pi$ to $0.97 \pi$ radians/second.

The proposed FODIs have very less PARE over $90 \%$ of Nyquist frequency range. Thus, these can be regarded as wideband FODIs. It is also verified that the proposed family of fractional order differintegrators outperforms all the existing FODs and FOIs over the entire Nyquist frequency range. These proposed fractional order differintegrators can be used in variety of practical applications as they have very less PARE and linear phase response over the entire frequency range.

\section{REFERENCES}

[1] M. Gupta, M. Jain, and B. Kumar, "Novel Class of Stable Wideband Recursive Digital Integrators and Differentiators," IET Signal Processing, vol.4, no.5, pp.560-566, 2010.

[2] M. Gupta, M. Jain, and B. Kumar, "Recursive Wideband Digital Integrator and Differentiator," International Journal of Circuit Theory and Applications, vol.39, no.7, pp.775-782, 2011.

[3] M. Gupta, M. Jain, and B. Kumar, "Linear Phase Second Order Recursive Digital Integrators and Differentiators," Radio Engineering, vol. 21, no. 2, pp. 712-717, 2012.

[4] M. Gupta, M. Jain, and B. Kumar, "Wideband Digital Integrator and Differentiator," IETE Journal of Research, vol. 58, no. 2, pp. 166 - 170, 2012.

[5] M. Jain, M. Gupta, and N. Jain, "Analysis and Design of Digital IIR Integrators and Differentiators using Minimax and Pole, Zero and Constant Optimization Methods," ISRN Electronics, vol. 2013, pp. 1 - 14, 2013.

[6] M. Jain, M. Gupta, and N. Jain, "The Design of the IIR Differintegrator and its Application in Edge Detection," Journal of Information Processing Systems, vol. 10, no. 2, pp. 223 - 239, 2014.

[7] M. Jain, M. Gupta, and N. Jain, "Design of Half Sample Delay Recursive Digital Integrators using Trapezoidal Integration Rule," International Journal of Signal \& Imaging Systems Engineering, vol. 9, no. 2, pp. 126 - 134, 2016.

[8] Y.Q Chen, B.M. Vinagre, and I. Podlubny, "Continued fraction expansion approaches to discretizing fractional order derivatives-an expository review," Nonlinear Dynamics, 2004, vol.38, no. 1-2, pp. 155-170.

[9] R.S. Barbosa, J.A.T. Machado, and I. M. Ferreira, "PoleZero Approximations Of Digital Fractional-Order Integrators And Differentiators Using Signal Modeling Techniques," IFAC Proceedings, vol. 38, no. 1, pp. 309314, 2005.

[10] C.C. Tseng, "Design of variable and adaptive fractional order differentiators," Signal Processing, vol.86, no.10, pp 2554-2566, 2006

[11] C.C. Tseng, "Series expansion design of variable fractional order integrator and differentiator using logarithm," Signal Processing, vol.88, no.9, pp. 22782292, 2008.

[12] Y. Ferdi "Computation of fractional order derivative and integral by power series expansion and signal modelling," Nonlinear Dynamics, vol. 46, no. 1-2, pp. 1-15, 2006.

[13] C.C. Tseng, and S.L. Lee "Design of Fractional Order Digital Differentiator Using Radial Basis Function," IEEE Transactions on circuits and systems-I: Regular papers, vol. 57, no. 7, pp. 1708-1718, 2010.

[14] B.T. Krishna, "Studies on fractional order differentiators and integrators: A survey," Signal Processing, vol. 91, pp. 386-426, 2011.

[15] M. Gupta, P. Varshney, and G.S. Visweswaran, "Digital fractional order differentiator and integrator models based on first order and higher order operators," International Journal of Circuit Theory and Applications, vol. 39, p. 461-474, 2011.

[16] M. Gupta, M. Jain, and N. Jain, "A new fractional order recursive digital integrator using continued fraction expansion," In the proceedings of 2010 IEEE India International Conference on Power Electronics (IICPE 2010), pp. 1-6, January 2011, India. 
[17] Y. LI, H. Sheng, and Y.Q. Chen, "On distributed order integrator / differentiator," Signal Processing, vol. 91, no. 5, pp. 1079-1084, 2011.

[18] F. Leulmi, and Y. Ferdi "An improvement of the rational approximation of the fractional operator $\mathrm{s}^{\alpha}$," Proceeding SIECPC 2011 (Electronics, Communications and Photonics Conference), pp 1 - 6, Saudi International, 2011

[19] M. Gupta, M. Jain, and B. Kumar, "Wideband digital integrator", In the proceedings of IEEE International Conference on Multimedia, Signal Processing and Communication Technologies (IMPACT 2009), pp. 107109, March 2009, India.

[20] N. Shrivastava, and P. Varshney, "Implementation of Carlson based Fractional Differentiators in Control of Fractional Order Plants," International Journal of Intelligent Systems and Applications, vol. 10, no. 9, pp. 66-74, 2018.

[21] F. T. Mohammed, M. A. Bahaaaldeen, A. S. Lobna, H. M. Ahmed, and G. R. Ahmed, "Fractional order integrator/differentiator: FPGA implementation and FOPID controller application," AEU - International Journal of Electronics and Communications, vol. 98, pp. 220-229, 2019.

[22] A. Ralston, and H.S. Wilf, Mathematical Methods for Digital Computers, Chapter 13. Wiley Publication, New York, 1960.

[23] W.H. Press, S.A Teukolsky, W.T. Vetterling, and B.P. Flannery, Numerical Recipes in $C$. The art of scientific computation. 2nd Edition. Cambridge University Press. Cambridge, 1992.

[24] E. Y. Remez, General Computational Methods of Chebyshev Approximations: The Problems with Linear Real Parameters, Books 1 and 2, Publishing House of the Academy of Science of the Ukrainian S.S.R. Kiev: English translation, AEC-TR-4491, United States Atomic Energy Commission, 1957.

[25] W.J. Cody, "A survey of practical rational and polynomial approximation of functions," SIAM Review, vol. 12, no. 3, pp. 400-423, 1970.

[26] M.A. Al-Alaoui, "Novel approach to designing digital differentiators," Electronics Letters, vol. 28, no. 15, pp. 1376-1378, 1992

\section{Authors' Profiles}

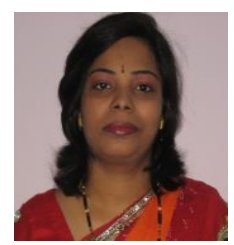

Madhu Jain is Associate Professor in Electronics \& Communication Engineering Department, Jaypee Institute of Information Technology, Noida, India. She received B.E. degree in Electronics and Communication Engineering from University of Rajasthan, M. Tech. degree in Signal Processing from University of Delhi and Ph. D. degree from Indian Institute of Technology, Delhi, India. Her major teaching and research interest include Signal Processing and Embedded System. She has co-authored 18 research papers in the above areas in various international journals and conferences.

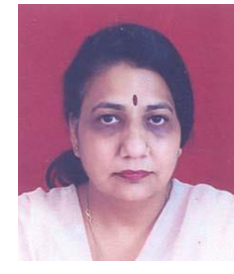

Mannesha Gupta is Professor in Electronics \& Communication Engineering Department of Netaji Subhas University of Technology, New Delhi, India. She received B.E. and M.E degree in Electronics and Communication Engineering from Government Engineering College, Jabalpur and $\mathrm{Ph}$. D. degree from Indian Institute of Technology, Delhi, India. Her major teaching and research interest include Switched Capacitors Circuits and Analog Signal processing. She has co-authored 95 research papers in the above areas in various international journals and conferences.

How to cite this paper: Madhu Jain, Maneesha Gupta, "Design of Fractional Order Recursive Digital Differintegrators using Different Approximation Techniques", International Journal of Intelligent Systems and Applications(IJISA), Vol.12, No.1, pp.33-42, 2020. DOI: 10.5815/ijisa.2020.01.04 